Article

\title{
Sodium Accumulation and Blood Capillary Rarefaction in the Skin Predispose Spontaneously Hypertensive Rats to Salt Sensitive Hypertension
}

\author{
Jan Šilhavý ${ }^{1, *}$, Petr Mlejnek ${ }^{1}$, Miroslava Šimáková ${ }^{1}$, František Liška ${ }^{1,2}$ D, Jan Kubovčiak ${ }^{3}$, Eva Sticová ${ }^{4,5}$ \\ and Michal Pravenec 1,2 iD
}

Citation: Šilhavý, J.; Mlejnek, P.; Šimáková, M.; Liška, F.; Kubovčiak, J.; Sticová, E.; Pravenec, M. Sodium Accumulation and Blood Capillary Rarefaction in the Skin Predispose Spontaneously Hypertensive Rats to Salt Sensitive Hypertension.

Biomedicines 2022, 10, 376. https:// doi.org/10.3390/biomedicines 10020376

Academic Editors: Guido Grassi and Pasquale Ambrosino

Received: 12 January 2022

Accepted: 2 February 2022

Published: 4 February 2022

Publisher's Note: MDPI stays neutral with regard to jurisdictional claims in published maps and institutional affiliations.

Copyright: (C) 2022 by the authors. Licensee MDPI, Basel, Switzerland. This article is an open access article distributed under the terms and conditions of the Creative Commons Attribution (CC BY) license (https:// creativecommons.org/licenses/by/ $4.0 /)$
1 Institute of Physiology, Czech Academy of Sciences, 14220 Prague, Czech Republic; Petr.Mlejnek@fgu.cas.cz (P.M.); Miroslava.Simakova@fgu.cas.cz (M.Š.); Frantisek.Liska@lf1.cuni.cz (F.L.); Michal.Pravenec@fgu.cas.cz (M.P.)

2 Institute of Biology and Medical Genetics, First Faculty of Medicine, Charles University, General University Hospital, 12800 Prague, Czech Republic

3 Laboratory of Genomics and Bioinformatics, Institute of Molecular Genetics, Czech Academy of Sciences, 14220 Prague, Czech Republic; kubovcij@img.cas.cz

4 Institute for Clinical and Experimental Medicine, 14021 Prague, Czech Republic; evsc@ikem.cz

5 Department of Pathology, Third Faculty of Medicine, Charles University, 10000 Prague, Czech Republic

* Correspondence: Jan.Silhavy@fgu.cas.cz

\begin{abstract}
Recent studies in humans and rats suggested that increased $\mathrm{Na}^{+}$storage in the skin without parallel water retention may predispose to salt-sensitive hypertension. In the current studies, we compared tissue $\mathrm{Na}^{+}$storage in salt sensitive spontaneously hypertensive rats (SHR) versus salt resistant normotensive Brown Norway (BN- $L x$ ) rats. After salt loading (10 days drinking $1 \% \mathrm{NaCl}$ solution), the SHR showed significant parallel increase in $\mathrm{Na}^{+}$-to-water as well as $\left(\mathrm{Na}^{+}+\mathrm{K}^{+}\right)$-to-water ratios suggesting increased storage of osmotically inactive $\mathrm{Na}^{+}$in the skin while no significant changes in skin electrolyte concentrations were observed in BN-Lx rats. SHR rats after salt treatment exhibited a nonsignificant decrease in skin blood capillary number (rarefaction) while BN-Lx rats showed significantly increased skin blood capillary density. Analysis of dermal gene expression profiles in BN-Lx rats after salt treatment showed significant up-regulation of genes involved in angiogenesis and proliferation of endothelial cells contrary to the SHR. Since the skin harbors most of the body's resistance vessels it is possible that blood capillary rarefaction may lead to increased peripheral resistance and salt sensitivity in the SHR.
\end{abstract}

Keywords: salt-sensitive hypertension; spontaneously hypertensive rat; skin; sodium; salt; blood pressure; capillary rarefaction; gene expression

\section{Introduction}

It has long been recognized that in some people substantially increasing dietary intake of salt increases blood pressure, whereas in others, increased salt intake has little or no effect on blood pressure. Although the blood pressure response to salt is a continuous variable and the trait of salt sensitivity, such as that of hypertension, is arbitrarily defined, it has been estimated that $30 \%$ to $50 \%$ of hypertensive humans are salt sensitive and approximately $25 \%$ of normotensive humans are salt sensitive [1,2]. Salt sensitivity confers an increased risk for the occurrence of hypertension and cardiovascular disease. Furthermore, pathophysiological mechanisms mediating salt sensitivity may contribute to the risk for cardiovascular disease beyond their effects on blood pressure [3]. Accordingly, the mechanisms of salt sensitivity continue to be studied intensively with the hope that better understanding of those mechanisms could lead to improved approaches to the prevention and treatment of salt-induced increases in blood pressure and cardiovascular disease. 
Recent studies in humans and rats provided evidence that $\mathrm{Na}^{+}$could be stored in the body, mainly in the skin, without a parallel water retention to buffer free extracellular $\mathrm{Na}^{+}[4,5]$. Some investigators have proposed that accumulation of $\mathrm{Na}^{+}$in skin tissue may influence blood pressure responses to changes in salt intake [6]. However, it is not known how $\mathrm{Na}^{+}$accumulation in the skin predisposes to salt-sensitive hypertension. It is possible that the amount of $\mathrm{Na}^{+}$stored in skin tissue may regulate blood pressure by influencing the extent to which a high salt intake affects blood volume and cardiac output, and vascular resistance, or both. For example, it is possible that increased accumulation of $\mathrm{Na}^{+}$in skin may affect the skin microcirculation and reduce skin blood capillary density. Skin blood capillary rarefaction, the reduction in the density of capillaries, has been associated with hypertension [7-9]. Capillary rarefaction is believed to mediate blood pressure changes by altering peripheral vascular resistance. He et al. [10] showed that in hypertensive humans, a modest reduction in salt intake increased dermal capillary density. The mechanisms whereby salt causes changes in the skin microcirculation remain unclear. The spontaneously hypertensive rats (SHR) are salt sensitive when compared to Brown Norway (BN- $L x$ ) rats [11], however, the role of tissue $\mathrm{Na}^{+}$accumulation in the pathogenesis of salt sensitivity was not analyzed in this animal model. In the current study, we tested the hypothesis that $\mathrm{Na}^{+}$accumulation in the skin may lead to blood capillary rarefaction and increased peripheral resistance and salt sensitivity in the SHR. In addition, we performed gene expression profiling in the skin to search for genes and pathways regulating dermal $\mathrm{Na}^{+}$ accumulation and angiogenesis.

\section{Materials and Methods}

\subsection{Animals}

We used 10-week-old males from SHR/OlaIpcr and BN-Lx/Cub strains (hereafter referred to as SHR and BN-Lx). The SHR/OlaIpcv (Rat Genome Database ID 631848) and BN-Lx/Cub (Rat Genome Database ID 61113) strains were obtained from the animal facility of the Institute of Physiology, Czech Academy of Sciences. The SHR/OlaIpcv strain was transferred to the Institute of Physiology from Olac, Ltd. Bicester, UK and is a substrain of the SHR/N inbred strain from NIH. BN-Lx/Cub is a substrain of the originally sequenced BN/NHsdMcwi strain (RGD ID 61498). In addition, it is a congenic strain carrying a segment of chromosome 8 from the PD/Cub (Polydactylous) strain with the $L x$ (Polydactly luxate syndrome) mutation, coded by the mutatnt Zbtb16 gene [12,13]. We used young adult rats because our earlier experiments showed that at this age BN-Lx and SHR strains clearly differ in their blood pressure and salt sensitivity [11]. Rats had free access to standard diet (Sniff ${ }^{\circledR}$ R-Z, Soest, Germany) containing $0.25 \% \mathrm{Na}^{+}$and water and they were held under humidity- and temperature-controlled conditions on a 12-12-h light-dark cycle. All animal experiments were conducted in compliance with the Animal Protection Law of the Czech Republic and were approved by the Ethics Committee of the Institute of Physiology, Czech Academy of Sciences, Prague (protocol code 71/2015).

\subsection{Experimental Protocol}

There were 4 experimental groups: Group $1-\mathrm{BN}-\operatorname{Lx}(\mathrm{N}=6)$ on regular chow and tap water; Group 2-BN-Lx $(\mathrm{N}=6)$ on regular chow and $1 \% \mathrm{NaCl}$ solution; Group 3-SHR $(\mathrm{N}=6)$ on regular chow and tap water and Group $4-\mathrm{SHR}(\mathrm{N}=6)$ on regular chow and $1 \% \mathrm{NaCl}$ solution. On day 1 , rats were placed into metabolic cages with standard chow and tap water. Every day 24-h aliquots of urine for $\mathrm{Na}^{+}$and $\mathrm{Cl}^{-}$were collected and fluid intake was recorded. On day 4, Group 2 (BN-Lx) and 4 (SHR) rats were given free access to $1 \% \mathrm{NaCl}$ solution instead of tap water. On day 15 , blood samples (for $\mathrm{Na}^{+}, \mathrm{K}^{+}$, and $\mathrm{Cl}^{-}$measurements) were taken from tail vein before the animals were killed by cervical dislocation. Samples of skin $(1 \mathrm{~cm} \times 2 \mathrm{~cm})$ were collected from the middle of subscapular region for determination of gene expression profiles (snap frozen in liquid nitrogen and stored at $-80^{\circ} \mathrm{C}$ ) and histological examination (stored in $4 \%$ formalin solution). 


\subsection{Tissue Electrolyte Determination}

Intestines and stomach were completely removed from rats to exclude remains of chow. The skins, gastrocnemius muscles, and carcasses (body without skin, intestines and stomach) were weighed to determined wet weight (WW) and then desiccated at $105^{\circ} \mathrm{C}$ for $36 \mathrm{~h}$ to determine dry weight (DW). The difference between $\mathrm{WW}$ and $\mathrm{DW}$ is considered as tissue water content. Ashing of carcasses was performed at $200{ }^{\circ} \mathrm{C}$ and $450{ }^{\circ} \mathrm{C}$ for $20 \mathrm{~h}$ at each temperature level, and the bones were sieved from the carcass ashes. Ashing of skins was performed at $200{ }^{\circ} \mathrm{C}$ and $450{ }^{\circ} \mathrm{C}$ for $10 \mathrm{~h}$ at each temperature level. Ashes were sent for analysis of $\mathrm{Na}^{+}$and $\mathrm{K}^{+}$concentrations by atomic adsorption spectrometry to ALS Czech Republic, Ltd. (Prague, Czech Republic). $\mathrm{Cl}^{-}$concentrations in the ashes were measured by titration with $0.1 \mathrm{~N}$ silver nitrate in the State Veterinary Institute (Prague, Czech Republic). Since the body weights of SHR and BN-Lx strains are significantly different, all chemical parameters in tissues are expressed as relative values corrected to dry weights and in the case of relative water content to wet weights. We also calculated $\mathrm{Na}^{+}$-to-water, $\mathrm{K}^{+}$to-water, and $\left(\mathrm{Na}^{+}+\mathrm{K}^{+}\right)$-to-water ratios. Parallel increases in skin $\mathrm{Na}^{+}$-to-water and skin $\left(\mathrm{Na}^{+}+\mathrm{K}^{+}\right)$-to-water ratios indicate increased $\mathrm{Na}^{+}$cation abundance relative to water and hence osmotically inactive $\mathrm{Na}^{+}$storage in the skin.

\subsection{Gene Expression Profiling}

We used RNAseq method for determination of gene expression profiles in the skin as described [14]. Library preparation was carried out with SENSE total RNA AEQ library prep kit for Illumina (PN A01107, Lexogene GmbH, Vienna, Austria). Library size distribution was evaluated on the Agilent 2100 Bioanalyzer using the High Sensitivity DNA Kit (Agilent Technologies Inc, Santa Clara, CA, USA). Libraries were sequenced on the Illumina NextSeq ${ }^{\circledR} 500$ instrument using 84 bp single-end configuration. Read quality was assessed by FastQC. For subsequent read processing, a bioinformatic pipeline nf-core/rnaseq version 1.4.2, was used. Individual steps included removing sequencing adaptors with Trim Galore, mapping to reference genome Rnor_6.0 (Ensembl annotation version 99) with HISAT2 and quantifying expression on gene level with featureCounts. Per gene mapped counts served as input for differential expression analysis using DESeq2 $\mathrm{R}$ Bioconductor package. Prior to the analysis, genes not expressed in at least two samples were discarded. Shrunken log2-fold changes using the adaptive shrinkage estimator were used for differential expression analysis. We supplied experimental model assuming sample treatment as main effect. Genes exhibiting minimal absolute $\log _{2}$-fold change value of 1 and statistical significance (adjusted $p$-value $<0.05$ ) between compared groups of samples were considered as differentially expressed (Figure S1). Gene set enrichment analysis was carried out using gene length bias aware algorithm implemented in goseq $\mathrm{R}$ Bioconductor package with KEGG pathways and GO terms data.

\subsection{Number of Blood and Lymph Capillaries}

Two full-thickness skin excisions taken from each animal were fixed in $4 \%$ formaldehyde and routinely processed by paraffin technique. Tissue sections $4 \mu \mathrm{m}$ thick were cut from each block and stained with hematoxylin and eosin (H\&E).

For immunohistochemical analysis, tissue sections ( $4 \mu \mathrm{m}$-thick) were pre-treated by heat mediated antigen retrieval and incubated with primary rabbit polyclonal antiCD31/PECAM-1 antibody (AP31323PU-N, Acris Antibodies GmbH, Herford, Germany, dilution 1:1000) to detect vascular endothelia, and with primary mouse monoclonal antibody anti-Podoplanin (clone LF3/B7/D5B27, NB110-96423, Novus Biologicals, Cambridge, UK, dilution 1:200) to detect lymphatic vessels. The primary antibodies were applied overnight at $4{ }^{\circ} \mathrm{C}$. The CD31 antibody was detected by biotinylated goat anti-rabbit IgG $(\mathrm{H}+\mathrm{L})$ (BA-1000-1.5, Vector Laboratories, Burlingame, CA, USA, dilution 1:200), after which the sections were incubated with VECTASTAIN ${ }^{\circledR}$ Elite ABC-HRP Reagent, Peroxidase, R.T.U. (PK-7100, Vector Laboratories) for $30 \mathrm{~min}$. The Simple Stain MAX PO (MULTI) Universal Immuno-peroxidase Polymer anti-mouse, anti-rabbit Histofine (414152F, Nichirei 
Biosciences, Tokyo, Japan) was used to detect the primary anti-podoplanin antibody. Finally, the sections were stained with the Dako Liquid DAB Substrate-Chromogen System (K3468, Dako, Glostrup, Denmark) and counterstained with Harris's haematoxylin.

For quantifications, 10 non-overlapping consecutive fields at magnification $\times 40$ with representation of full-thickness dermal compartment were captured from each tissue section using a microscope Olympus BX51 and a camera MICROPIX. Positively stained dermal blood vessels and lymphatic vessels were counted in each field using Micropix v.2.0.4.0 image processing software (Micropix, Ltd., Chichester, UK). The results were expressed as the mean microvessel count with standard deviation.

\subsection{Statistical Analysis}

Results are expressed as means \pm S.E.M. SigmaPlot ${ }^{\circledR} 12$ software package was used for two-way ANOVA to test for strain $x$ salt interactions. For variables showing evidence of strain $\mathrm{x}$ salt interaction effects, we used Holm Sidak testing that adjusts for multiple comparisons to determine whether the effects of salt were significant in SHR versus BN-Lx rats.

\section{Results}

\subsection{Tissue Electrolyte Concentrations and Water Content}

Table 1 shows the body weights, tissue water content and electrolyte concentrations in all four experimental groups of rats. The SHR rats were significantly heavier when compared to $\mathrm{BN}-L x$ rats and therefore all electrolyte concentrations in tissues are expressed as relative values corrected to dry weights and in the case of relative water content to wet weights. Total body $\mathrm{Na}^{+}$significantly increased after salt loading in the SHR while no difference was observed between BN- $L x$ control versus BN- $L x$ salt-treated rats. There were no significant differences in total body $\mathrm{K}^{+}$between strains and treatment. On the other hand, when rats were treated with salt, the SHR showed significantly higher total body $\mathrm{Cl}^{-}$levels while BN-Lx rats showed no significant difference. Salt treatment had no effects on relative total body water (Table 1).

Table 1. Absolute wet weights and dry weights in various tissues and relative concentrations of $\mathrm{Na}^{+}$, $\mathrm{K}^{+}$, and $\mathrm{Cl}^{-}$in tissues from $\mathrm{BN}-L x$ and SHR rats given either tap water or $1 \%$ saline for drinking.

\begin{tabular}{|c|c|c|c|c|c|c|c|}
\hline \multirow[b]{2}{*}{ Traits } & \multirow{2}{*}{$\begin{array}{l}\text { BN-Lx } \\
\text { Control }\end{array}$} & \multirow{2}{*}{$\begin{array}{l}\text { BN-Lx } \\
\text { Salt }\end{array}$} & \multirow{2}{*}{$\begin{array}{c}\text { SHR } \\
\text { Control }\end{array}$} & \multirow{2}{*}{$\begin{array}{l}\text { SHR } \\
\text { Salt }\end{array}$} & \multicolumn{3}{|c|}{ Source of Variation $(p)$} \\
\hline & & & & & Strain & Salt & $\begin{array}{c}\text { Strain } \times \text { Salt } \\
\text { Interaction }\end{array}$ \\
\hline $\begin{array}{c}\text { Body WW } \\
\text { (skin WW + Carcass } \\
\text { WW) (g) }\end{array}$ & $228 \pm 10$ & $209 \pm 4$ & $308 \pm 3$ & $303 \pm 2$ & $<0.001$ & 0.067 & 0.253 \\
\hline $\begin{array}{c}\text { Body DW }(\mathrm{g}) \\
(\text { Carcass DW + Skin } \\
\text { DW })\end{array}$ & $76 \pm 3$ & $71 \pm 3$ & $109 \pm 1$ & $106 \pm 2$ & $<0.001$ & 0.102 & 0.664 \\
\hline Carcass WW (g) & $186 \pm 8$ & $168 \pm 3$ & $235 \pm 1$ & $228 \pm 2$ & $<0.001$ & 0.032 & 0.246 \\
\hline Carcass DW (g) & $58 \pm 2$ & $53 \pm 2$ & $77 \pm 1$ & $74 \pm 2$ & $<0.001$ & 0.050 & 0.571 \\
\hline Carcass water $(\mathrm{g})$ & $128 \pm 6$ & $115 \pm 1$ & $158 \pm 1$ & $155 \pm 1$ & $<0.001$ & 0.036 & 0.168 \\
\hline $\begin{array}{l}\text { Carcass relative water } \\
(\mathrm{mL} / \mathrm{g} W W)\end{array}$ & $0.687 \pm 0.003$ & $0.685 \pm 0.007$ & $0.674 \pm 0.002$ & $0.678 \pm 0.004$ & 0.046 & 0.788 & 0.438 \\
\hline Skin WW (g) & $43 \pm 1$ & $41 \pm 1$ & $72 \pm 2$ & $74 \pm 1$ & $<0.001$ & 0.964 & 0.428 \\
\hline Skin DW (g) & $18 \pm 2$ & $18 \pm 4$ & $32 \pm 4$ & $32 \pm 4$ & $<0.001$ & 0.752 & 0.986 \\
\hline Skin water $(\mathrm{g})$ & $24 \pm 1$ & $23 \pm 1$ & $40 \pm 2$ & $41 \pm 1$ & $<0.001$ & 0.843 & 0.230 \\
\hline $\begin{array}{l}\text { Skin relative water } \\
(\mathrm{mL} / \mathrm{g} W W)\end{array}$ & $0.571 \pm 0.006$ & $0.565 \pm 0.009$ & $0.552 \pm 0.006$ & $0.563 \pm 0.009$ & 0.204 & 0.770 & 0.310 \\
\hline
\end{tabular}


Table 1. Cont

\begin{tabular}{|c|c|c|c|c|c|c|c|}
\hline \multirow[b]{2}{*}{ Traits } & \multirow[b]{2}{*}{$\begin{array}{l}\text { BN-Lx } \\
\text { Control }\end{array}$} & \multirow[b]{2}{*}{$\begin{array}{l}\text { BN-Lx } \\
\text { Salt }\end{array}$} & \multirow[b]{2}{*}{$\begin{array}{c}\text { SHR } \\
\text { Control }\end{array}$} & \multirow[b]{2}{*}{$\begin{array}{l}\text { SHR } \\
\text { Salt }\end{array}$} & \multicolumn{3}{|c|}{ Source of Variation $(p)$} \\
\hline & & & & & Strain & Salt & $\begin{array}{c}\text { Strain } \times \text { Salt } \\
\text { Interaction }\end{array}$ \\
\hline rTBW (mL/g WW) & $1.258 \pm 0.009$ & $1.250 \pm 0.015$ & $1.226 \pm 0.005$ & $1.241 \pm 0.013$ & 0.108 & 0.765 & 0.329 \\
\hline Bone ash (g) & $7.6 \pm 0.3$ & $7.1 \pm 0.1$ & $8.6 \pm 0.2$ & $8.2 \pm 0.1$ & $<0.001$ & 0.059 & 0.982 \\
\hline $\begin{array}{c}\mathrm{rTB} \mathrm{Na} \\
(\mathrm{mmol} / \mathrm{g} \mathrm{DW})\end{array}$ & $0.220 \pm 0.005$ & $0.213 \pm 0.011$ & $0.192 \pm 0.005$ & $0.239 \pm 0.011$ \# & 0.938 & 0.045 & 0.013 \\
\hline $\mathrm{rTB} \mathrm{K}^{+}(\mathrm{mmol} / \mathrm{g} \mathrm{DW})$ & $0.293 \pm 0.010$ & $0.277 \pm 0.018$ & $0.246 \pm 0.007$ & $0.263 \pm 0.010$ & 0.030 & 0.974 & 0.202 \\
\hline $\begin{array}{c}\mathrm{rTB} \mathrm{Cl} \\
(\mathrm{mmol} / \mathrm{g} \mathrm{DW})\end{array}$ & $0.110 \pm 0.027$ & $0.072 \pm 0.005$ & $0.088 \pm 0.011$ & $0.123 \pm 0.008 \ddagger$ & 0.376 & 0.934 & 0.040 \\
\hline $\begin{array}{l}\text { rCarcass Na+ } \\
(\mathrm{mmol} / \mathrm{g} \mathrm{DW})\end{array}$ & $0.093 \pm 0.002$ & $0.089 \pm 0.006$ & $0.077 \pm 0.003^{\dagger}$ & $0.090 \pm 0.002^{\#}$ & 0.070 & 0.220 & 0.036 \\
\hline $\begin{array}{c}\text { rCarcass K+ } \\
(\mathrm{mmol} / \mathrm{g} \mathrm{DW})\end{array}$ & $0.197 \pm 0.009$ & $0.166 \pm 0.009$ & $0.158 \pm 0.005$ & $0.171 \pm 0.004$ & 0.026 & 0.726 & 0.091 \\
\hline $\begin{array}{l}\mathrm{rCarcass} \mathrm{Cl}^{-} \\
(\mathrm{mmol} / \mathrm{g} \mathrm{DW})\end{array}$ & $0.015 \pm 0.001$ & $0.013 \pm 0.001$ & $0.015 \pm 0.001$ & $0.021 \pm 0.001$ \# & 0.004 & 0.026 & 0.002 \\
\hline $\begin{array}{c}\mathrm{rSkin} \mathrm{Na}^{+} \\
(\mathrm{mmol} / \mathrm{g} \mathrm{DW})\end{array}$ & $0.127 \pm 0.002$ & $0.124 \pm 0.005$ & $0.116 \pm 0.002$ & $0.149 \pm 0.009$ \# & 0.268 & 0.025 & 0.012 \\
\hline $\begin{array}{c}\text { rSkin } \mathrm{K}^{+} \\
(\mathrm{mmol} / \mathrm{g} \mathrm{DW})\end{array}$ & $0.096 \pm 0.001$ & $0.100 \pm 0.005$ & $0.088 \pm 0.002$ & $0.092 \pm 0.006$ & 0.078 & 0.422 & 0.950 \\
\hline $\begin{array}{c}\mathrm{rSkin} \mathrm{Cl}^{-} \\
(\mathrm{mmol} / \mathrm{g} \mathrm{DW})\end{array}$ & $0.070 \pm 0.009$ & $0.059 \pm 0.004$ & $0.073 \pm 0.010$ & $0.102 \pm 0.007 \ddagger$ & 0.370 & 0.978 & 0.037 \\
\hline $\begin{array}{c}\text { rBone } \mathrm{Na}^{+} \\
(\mathrm{mmol} / \mathrm{g} \text { carcass DW) }\end{array}$ & $0.068 \pm 0.001$ & $0.072 \pm 0.001$ & $0.064 \pm 0.003$ & $0.065 \pm 0.001$ & 0.173 & 0.775 & 0.156 \\
\hline $\begin{array}{c}\mathrm{rBone}^{+} \\
(\mathrm{mmol} / \mathrm{g} \text { carcass DW) }\end{array}$ & $0.055 \pm 0.004$ & $0.070 \pm 0.005$ & $0.040 \pm 0.003$ & $0.038 \pm 0.001$ & 0.003 & 0.056 & 0.234 \\
\hline $\begin{array}{c}\mathrm{rBone} \mathrm{Cl}^{-} \\
(\mathrm{mmol} / \mathrm{g} \text { carcass DW) }\end{array}$ & $0.019 \pm 0.001$ & $\begin{array}{c}0.020 \pm 0.001 \\
*\end{array}$ & $0.018 \pm 0.001^{+}$ & $0.019 \pm 0.001 \ddagger$ & $<0.001$ & 0.103 & 0.046 \\
\hline Serum $\mathrm{Na}^{+}(\mathrm{mmol} / \mathrm{L})$ & $141.9 \pm 0.3$ & $143.2 \pm 0.6$ & $143.2 \pm 0.6$ & $143.4 \pm 0.8$ & 0.245 & 0.265 & 0.381 \\
\hline Serum $\mathrm{K}^{+}(\mathrm{mmol} / \mathrm{L})$ & $5.2 \pm 0.1$ & $5.0 \pm 0.1$ & $5.2 \pm 0.1$ & $4.9 \pm 0.1^{\ddagger}$ & 0.524 & 0.009 & 0.294 \\
\hline Serum $\mathrm{Cl}^{-}(\mathrm{mmol} / \mathrm{L})$ & $105.2 \pm 0.8$ & $106.6 \pm 0.7$ & $103.7 \pm 0.7$ & $105.2 \pm 0.9$ & 0.094 & 0.104 & 0.935 \\
\hline
\end{tabular}

Two-way ANOVA results: $p$ values of statistical significance for effects strain, salt treatment and strain $\mathrm{x}$ salt interaction (the effects of salt are different in $B N-L x$ versus $S H R$ rats). For pairwise multiple comparison procedures Holm Sidak testing was used: * denotes significant $p<0.05$ effects of salt within BN- $L x$ rats; ${ }^{\#}$ denotes significant $p<0.05$ effects of salt within SHR rats; ${ }^{\dagger}$ denotes $p<0.05$ significant differences between strains on normal salt intake; $\ddagger$ denotes $p<0.05$ significant differences between strains on high salt intake.

Skin $\mathrm{Na}^{+}$increased after salt treatment in the SHR but not in BN-Lx rats (Figure $1 \mathrm{~A}$, Table 1). There were no significant differences in skin $\mathrm{K}^{+}$concentrations both between strains and salt treatment. After treatment with salt, SHR rats showed significantly higher $\mathrm{Cl}^{-}$levels in the skin while BN-Lx rats showed no significant difference. Skin was the major depot for $\mathrm{Na}^{+}$accumulation when skin of $\mathrm{BN}-L x$ and SHR rats contained $32 \%$ and $42 \%$ of total body $\mathrm{Na}^{+}$, respectively.

Carcass $\mathrm{Na}^{+}$increased in SHR rats after salt treatment but not in BN- $L x$ rats. Carcass $\mathrm{K}^{+}$levels in BN-Lx rats were not significantly different from the SHR. On the other hand, $\mathrm{Cl}^{-}$carcass concentrations increased in the SHR after salt treatment while no significant differences were observed between BN- $L x$ control and experimental groups (Table 1). 
A.

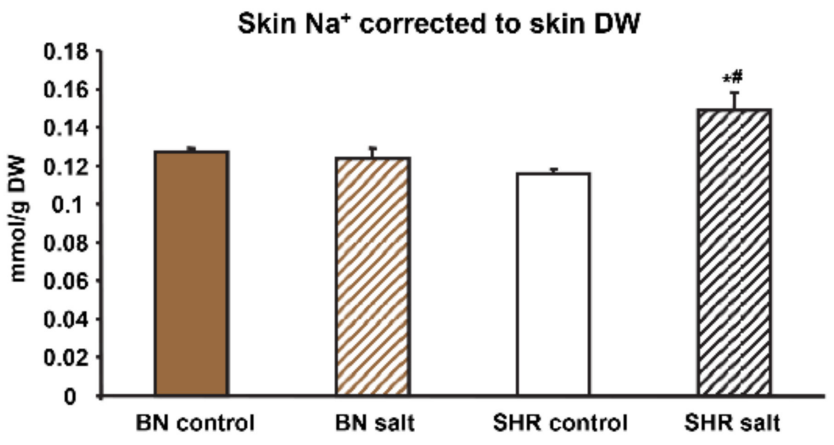

B.

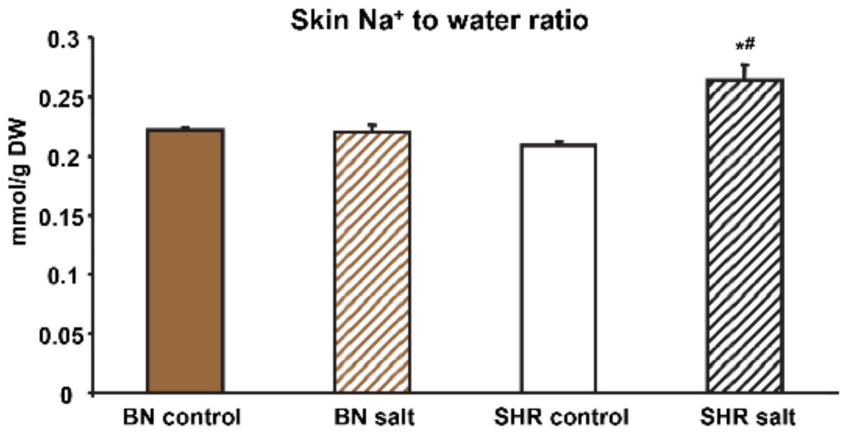

C.

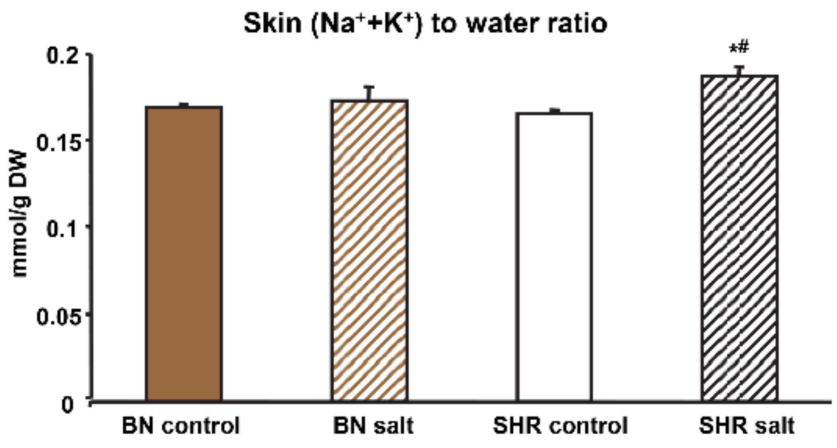

Figure 1. Electrolyte concentrations in the skin of SHR and BN-Lx strains. (A) Salt treatment of SHR rats was associated with significant accumulation of relative (corrected to dry weight) $\mathrm{Na}^{+}$in the skin. (B) SHR rats treated with salt exhibited significantly increased $\mathrm{Na}^{+}$-to-water ratio (B) and also $\left(\mathrm{Na}^{+}+\mathrm{K}^{+}\right)$-to-water ratio $(\mathrm{C})$. Parallel increase of $\mathrm{Na}^{+}$-to-water ratio and $\left(\mathrm{Na}^{+}+\mathrm{K}^{+}\right)$-to-water ratio indicates higher amount of osmotically inactive $\mathrm{Na}^{+}$storage. * denotes two-way ANOVA significant strain $\times$ salt interaction effects $(p<0.05)$, ${ }^{\#}$ denotes significantly higher value in the SHR after salt treatment $(p<0.05)$.

No significant differences in muscle electrolyte concentrations and water content were observed between strains and salt treatment (Table 1).

No significant differences were observed in bone $\mathrm{Na}^{+}$concentrations. On the other hand, BN-Lx rats showed significantly increased bone $\mathrm{K}^{+}$after salt treatment and had higher $\mathrm{K}^{+}$levels when compared to SHR rats. There were small but significant differences in bone $\mathrm{Cl}^{-}$concentrations among the groups (Table 1).

\section{2. $\mathrm{Na}^{+}$-to-Water, $\mathrm{K}^{+}$-to-Water and $\left(\mathrm{Na}^{+}+\mathrm{K}^{+}\right)$-to-Water Ratios}

Table 2 shows the relationships between $\mathrm{Na}^{+}, \mathrm{K}^{+}$, and the sum of the two cations (total effective osmolytes) with total body water. The ratio of total body $\mathrm{Na}^{+}$per total body 
water was increased by salt only in the SHR while remained unchanged in BN-Lx rats. This increased ratio of total body $\mathrm{Na}^{+}$-to-water was mainly due to higher storage of $\mathrm{Na}^{+}$in the skin of SHR rats because no significant differences were observed in carcass, muscle and bone $\mathrm{Na}^{+}$-to-water ratios (Figure $1 \mathrm{~B}$, Table 2).

Table 2. $\mathrm{Na}^{+}$-to-water, $\mathrm{K}^{+}$-to-water, and $\left(\mathrm{Na}^{+}+\mathrm{K}^{+}\right)$-to-water ratios.

\begin{tabular}{|c|c|c|c|c|c|c|c|}
\hline Traits & $\begin{array}{l}\text { BN-Lx } \\
\text { Control }\end{array}$ & BN-Lx Salt & SHR Control & SHR Salt & \multicolumn{3}{|c|}{ Source of Variation $(p)$} \\
\hline & & & & & Strain & Salt & $\begin{array}{c}\text { Strain } \times \\
\text { Salt } \\
\text { Interaction }\end{array}$ \\
\hline \multicolumn{8}{|c|}{$\mathrm{Na}^{+}$-to-water ratio, $\mathrm{mmol} / \mathrm{mL}$} \\
\hline $\mathrm{TB} \mathrm{Na}^{+} / \mathrm{TB} \mathrm{W}$ & $0.051 \pm 0.001$ & $0.050 \pm 0.001$ & $0.049 \pm 0.002$ & $0.058 \pm 0.001^{\# \ddagger}$ & 0.032 & 0.005 & 0.002 \\
\hline $\begin{array}{c}\text { Carcass } \mathrm{Na}^{+} / \\
\text {Carcass W }\end{array}$ & $0.042 \pm 0.001$ & $0.041 \pm 0.001$ & $0.037 \pm 0.002^{\dagger}$ & $0.043 \pm 0.001$ \# & 0.229 & 0.132 & 0.017 \\
\hline Skin $\mathrm{Na}^{+} /$Skin $\mathrm{W}$ & $0.095 \pm 0.001$ & $0.096 \pm 0.001$ & $0.094 \pm 0.001$ & $0.115 \pm 0.004^{\# \ddagger}$ & 0.003 & $<0.001$ & 0.001 \\
\hline Bone $\mathrm{Na}^{+} / \mathrm{TB} \mathrm{W}$ & $0.026 \pm 0.001$ & $0.028 \pm 0.001$ & $0.026 \pm 0.002$ & $0.024 \pm 0.003$ & 0.146 & 0.900 & 0.089 \\
\hline \multicolumn{8}{|c|}{$\mathrm{K}^{+}$-to-water ratio, $\mathrm{mmol} / \mathrm{mL}$} \\
\hline $\mathrm{TB} \mathrm{K}^{+} / \mathrm{TB} \mathrm{W}$ & $0.087 \pm 0.002$ & $0.081 \pm 0.003$ & $0.073 \pm 0.008^{\dagger}$ & $0.079 \pm 0.004$ & 0.005 & 0.924 & 0.018 \\
\hline Carcass $\mathrm{K}^{+} /$Carcass $\mathrm{W}$ & $0.090 \pm 0.003$ & $0.082 \pm 0.004 *$ & $0.074 \pm 0.005^{\dagger}$ & $0.081 \pm 0.001$ & 0.011 & 0.804 & 0.015 \\
\hline Skin $\mathrm{K}^{+} /$Skin $\mathrm{W}$ & $0.073 \pm 0.001$ & $0.077 \pm 0.002 *$ & $0.071 \pm 0.001$ & $0.070 \pm 0.002 \ddagger$ & 0.010 & 0.337 & 0.046 \\
\hline Bone $\mathrm{K}^{+} / \mathrm{TB} \mathrm{W}$ & $0.020 \pm 0.001$ & $0.027 \pm 0.003 *$ & $0.016 \pm 0.001$ & $0.014 \pm 0.001 \ddagger$ & $<0.001$ & 0.068 & 0.019 \\
\hline \multicolumn{8}{|c|}{$\left(\mathrm{Na}^{+}+\mathrm{K}^{+}\right)$-to-water ratio, $\mathrm{mmol} / \mathrm{mL}$} \\
\hline $\mathrm{TB}\left(\mathrm{Na}^{+}+\mathrm{K}^{+}\right) / \mathrm{TB} \mathrm{W}$ & $0.138 \pm 0.003$ & $0.131 \pm 0.004$ & $0.124 \pm 0.004^{\dagger}$ & $0.137 \pm 0.001^{\#}$ & 0.293 & 0.397 & 0.016 \\
\hline $\begin{array}{c}\text { Carcass }\left(\mathrm{Na}^{+}+\mathrm{K}^{+}\right) / \\
\text {Carcass W }\end{array}$ & $0.132 \pm 0.004$ & $0.122 \pm 0.005$ & $0.114 \pm 0.005^{\dagger}$ & $0.124 \pm 0.001$ & 0.064 & 0.958 & 0.035 \\
\hline Skin $\left(\mathrm{Na}^{+}+\mathrm{K}^{+}\right) /$Skin $\mathrm{W}$ & $0.168 \pm 0.002$ & $0.172 \pm 0.008$ & $0.165 \pm 0.002$ & $0.186 \pm 0.005$ \# & 0.085 & 0.002 & 0.018 \\
\hline Bone $\left(\mathrm{Na}^{+}+\mathrm{K}^{+}\right) / \mathrm{TB} \mathrm{W}$ & $0.046 \pm 0.001$ & $0.054 \pm 0.003 *$ & $0.042 \pm 0.002$ & $0.039 \pm 0.001 \ddagger$ & 0.002 & 0.226 & 0.025 \\
\hline & $\begin{array}{l}\text { Two-way } \\
\text { interactio } \\
\text { Holm Sid } \\
p<0.05 \mathrm{e} \\
\text { intake; }{ }^{\ddagger}\end{array}$ & $\begin{array}{l}\text { NOVA results: } p \\
\text { he effects of salt a } \\
\text { testing was used: } \\
\text { cts of salt within } \\
\text { lotes } p<0.05 \text { sign }\end{array}$ & $\begin{array}{l}\text { des of statistical s } \\
\text { ifferent in BN- } L x \\
\text { enotes significant } \\
\text { rats; }{ }^{+} \text {denotes } p \\
\text { ant differences be }\end{array}$ & $\begin{array}{l}\text { nificance for effect } \\
\text { sus } \mathrm{SHR} \text { rats). For } \\
<0.05 \text { effects of sal } \\
0.05 \text { significant di } \\
\text { een strains on high }\end{array}$ & $\begin{array}{l}\text { rain, sa } \\
\text { wise m } \\
\text { thin BI } \\
\text { ences b } \\
\text { It intak }\end{array}$ & $\begin{array}{l}\text { reatmen } \\
\text { iple comp } \\
x \text { rats; }{ }^{\#} \\
\text { veen stra }\end{array}$ & $\begin{array}{l}\text { d strain } \times \text { sa } \\
\text { son procedure } \\
\text { otes significar } \\
\text { on normal sa }\end{array}$ \\
\hline
\end{tabular}

Total body $\mathrm{K}^{+}$-to-water ratio was higher in $\mathrm{BN}-L x$ rats when compared to $\mathrm{SHR}$ rats on high salt intake. Carcass $\mathrm{K}^{+}$-to-water ratios was reduced while skin and bone $\mathrm{K}^{+}$-to-water ratios after salt were increased in BN-Lx rats but not in SHR rats (Table 2).

Salt treatment increased total body $\left(\mathrm{Na}^{+}+\mathrm{K}^{+}\right)$-to-water ratio in SHR rats but not in $\mathrm{BN}-L x$ rats which was mainly due to significant increase in skin $\left(\mathrm{Na}^{+}+\mathrm{K}^{+}\right)$-to-water ratio when compared to BN-Lx rats (Figure $1 C$ ). On the other hand, bone $\left(\mathrm{Na}^{+}+\mathrm{K}^{+}\right)$-to-water ratio was increased after salt in $\mathrm{BN}-L x$ rats but not in SHR rats. No significant differences in muscle $\left(\mathrm{Na}^{+}+\mathrm{K}^{+}\right)$-to-water ratio were observed between strains and salt treatment (Table 2 ). Parallel increases in $\mathrm{Na}^{+}$-to-water and $\left(\mathrm{Na}^{+}+\mathrm{K}^{+}\right)$-to-water ratios indicate increased $\mathrm{Na}^{+}$ cation abundance relative to water and hence osmotically inactive $\mathrm{Na}^{+}$storage in the skin of SHR rats.

\subsection{Fluid Ingestion and Cumulative $\mathrm{Na}^{+}$Balance}

Figure 2 shows fluid ingestion and cumulative $\mathrm{Na}^{+}$balance in SHR and BN-Lx rats drinking ad libitum either tap water or salt solution. As can be seen, on high salt diet the SHR ingested significantly more salt solution and exhibited significantly higher cumulative $\mathrm{Na}^{+}$balance when compared to BN-Lx rats. These differences remained significant when 
the ingested fluid was corrected to body weight (data not shown). These observations suggest that increased $\mathrm{Na}^{+}$accumulation in the skin of SHR rats might be due to increased salt ingestion.

\section{A. Fluid ingestion}
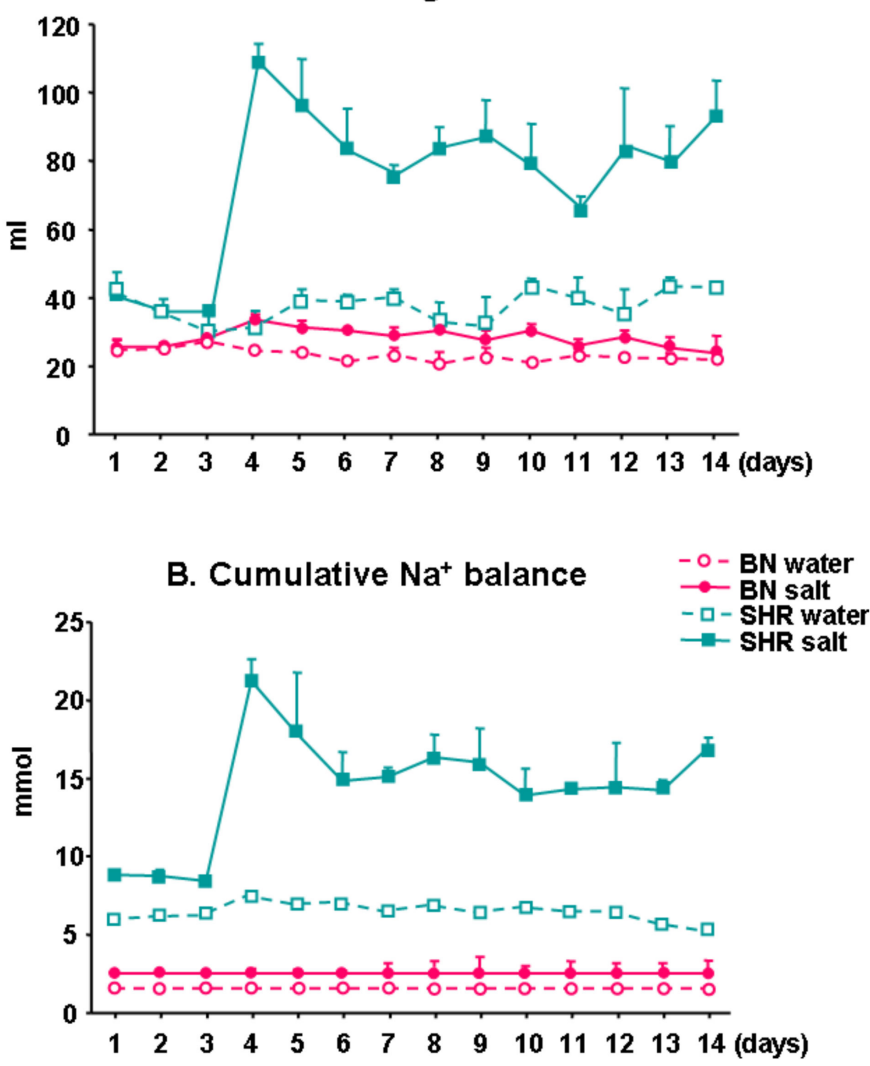

Figure 2. Fluid ingestion (A) and cumulative $\mathrm{Na}^{+}$balance (B). On day 4, experimental groups of $\mathrm{BN}-L x$ and SHR rats were given free access to $1 \% \mathrm{NaCl}$ solution while control BN-Lx and SHR stayed on tap water. The SHR rats ingested significantly more $\mathrm{NaCl}$ solution when compared to $\mathrm{BN}-L x$ rats and exhibited significantly increased cumulative $\mathrm{Na}^{+}$balance.

\subsection{Skin Blood and Lymph Capillary Density}

We measured number of blood capillaries in the skin of BN-Lx and SHR rats on normal or high salt intake. Two-way ANOVA showed significant strain $x$ salt interaction effects on blood capillary number in the skin. As can be seen in Figure $3 A$, BN- $L x$ rats after salt treatment exhibited significantly increased skin blood capillary number contrary to SHR rats. On the hand, we observed no significant differences in the number of lymph capillaries between BN-Lx and SHR strains and between control and high salt intake (Figure 3B). Figure 4 shows representative histological images of blood and lymphatic capillaries.

\subsection{Gene Expression Profiles in the Skin}

To search for molecular mechanisms responsible for differences in $\mathrm{Na}^{+}$accumulation in the skin and salt-dependent hypertension, we performed analysis of gene expression profiles using RNAseq method in response to salt loading in BN- $L x$ and SHR strains (Tables 3 and 4). Gene set enrichment analysis (GSEA) on biological processes (BP) in $\mathrm{BN}-L x$ rats identified as the most significantly up-regulated genes involved in regulation of angiogenesis and proliferation of endothelial cells (Table 3). On the other hand, in the SHR after salt treatment, GSEA on BP identified as the most significantly up-regulated genes involved mainly in regulation of keratinocyte differentiation and epidermis development, negative regulation of peptidase activity and innate immunity (Table 4). 
A.

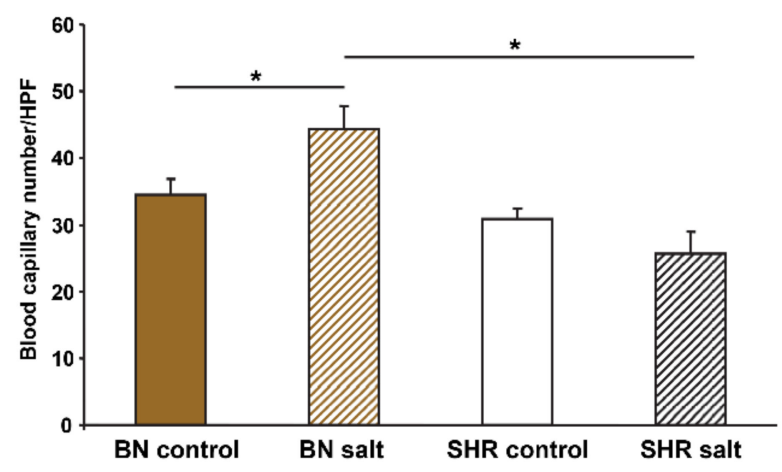

B.

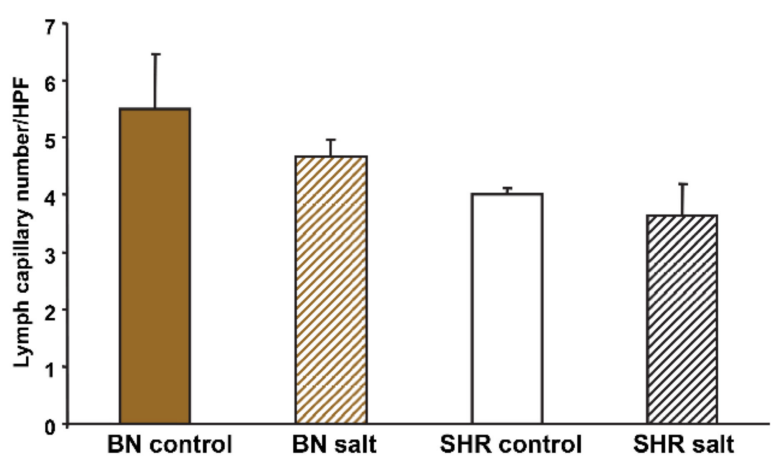

Figure 3. Skin blood and lymph capillary number in BN-Lx and SHR rats drinking either tap water (controls) or $1 \% \mathrm{NaCl}$ solution (salt) for 10 days. (A) Two-way ANOVA showed significant strain $\times$ salt interaction effects $(p=0.026)$. BN- $L x$ rats exhibited significantly increased blood capillary number after salt treatment when compared to BN-Lx controls and to SHR treated with salt. (B) No significant differences by two-way ANOVA were observed in lymph capillary density between strains and treatments. ${ }^{*}$ denotes $p<0.05$.

A.

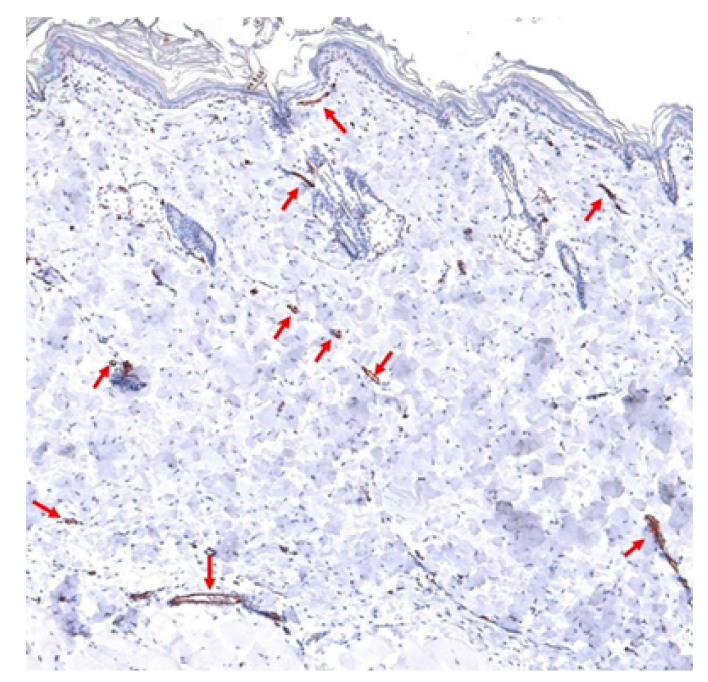

B.

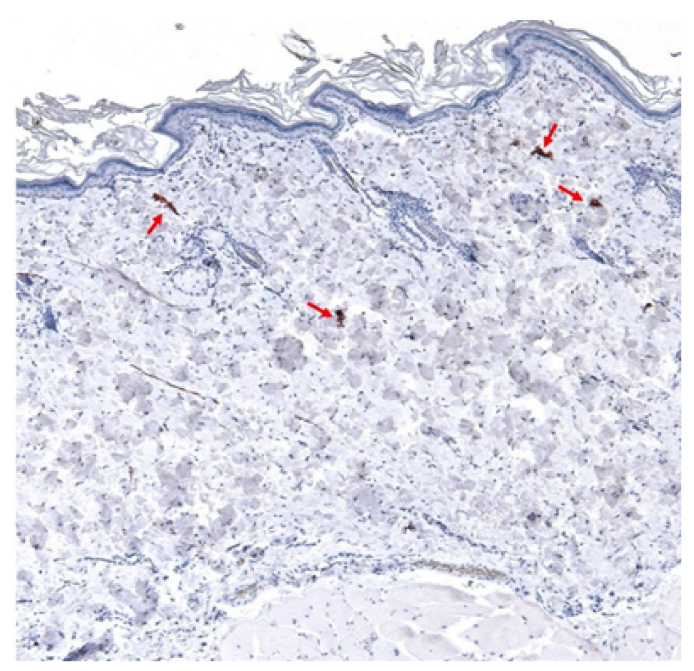

Figure 4. Representative images of skin blood and lymphatic capillaries. (A). Anti-CD31/PECAM-1 immunohistochemistry. The antibody stains endothelial lining of dermal blood vessels (arrows). Original magnification $\times 40$. (B). Anti-podoplanin immunohistochemistry. Primary antibody highlights dermal lymphatic vessels (arrows). Original magnification $\times 40$. 
Table 3. Differentially expressed genes from biological processes (BP) identified by Gene set enrichment analysis (GSEA) in $\mathrm{BN}-L x$ rats treated with $1 \% \mathrm{NaCl}$ drinking solution versus control $\mathrm{BN}-L x$ rats drinking tap water.

\begin{tabular}{|c|c|c|c|}
\hline Symbol & Name & $\log \mathrm{FC}$ & Adjusted $p$ Value \\
\hline \multicolumn{4}{|c|}{ BP: Positive regulation of angiogenesis, $p=8.7 \times 10^{-7}$} \\
\hline Ereg & epiregulin & 3.39 & 0.0018 \\
\hline Vegfd & vascular endothelial growth factor D & 1.60 & 0.0075 \\
\hline Angpt4 & angiopoietin 4 & 2.86 & 0.0018 \\
\hline Ccl11 & C-C motif chemokine ligand 11 & 2.06 & 0.0067 \\
\hline Sfrp2 & secreted frizzled-related protein 2 & 3.80 & 0.00015 \\
\hline F3 & coagulation factor III, tissue factor & 2.04 & 0.000027 \\
\hline C6 & complement C6 & 2.05 & 0.0073 \\
\hline \multicolumn{4}{|c|}{ BP: Positive regulation of endothelial cell proliferation, $p=1.30 \times 10^{-4}$} \\
\hline Vegfd & vascular endothelial growth factor D & 1.60 & 0.0075 \\
\hline Ccl11 & C-C motif chemokine ligand 11 & 2.06 & 0.0067 \\
\hline F3 & coagulation factor III, tissue factor & 2.04 & 0.000027 \\
\hline Wnt2 & Wnt family member 2 & 2.30 & 0.0049 \\
\hline \multicolumn{4}{|c|}{ BP: Complement activation, $p=1.90 \times 10^{-5}$} \\
\hline C6 & complement C6 & 2.05 & 0.0073 \\
\hline Cfh & complement factor $\mathrm{H}$ & 1.84 & 0.025 \\
\hline C7 & complement C7 & 0.0001 & 0.030 \\
\hline \multicolumn{4}{|c|}{ BP: Cellular response to interferon-gamma, $p=4.34 \times 10^{-4}$} \\
\hline Ccl11 & C-C motif chemokine ligand 11 & 2.06 & 0.0067 \\
\hline$M r c 1$ & mannose receptor, $\mathrm{C}$ type 1 & 1.59 & 0.029 \\
\hline Ccl6 & chemokine (C-C motif) ligand 6 & 2.12 & 0.0056 \\
\hline Cfh & complement factor $\mathrm{H}$ & 1.84 & 0.025 \\
\hline \multicolumn{4}{|c|}{ BP: Cellular response to interleukin-1, $p=5.84 \times 10^{-4}$} \\
\hline Smpd3 & sphingomyelin phosphodiesterase 3 & 2.35 & 0.0049 \\
\hline Ccl11 & C-C motif chemokine ligand 11 & 2.06 & 0.0067 \\
\hline Fn1 & fibronectin 1 & 2.59 & 0.00051 \\
\hline Ccl6 & chemokine (C-C motif) ligand 6 & 2.12 & 0.0056 \\
\hline \multicolumn{4}{|c|}{ BP: Chemokine-mediated signaling pathway, $p=7.25 \times 10^{-4}$} \\
\hline Cor1 & $\mathrm{C}-\mathrm{C}$ motif chemokine receptor 1 & 2.19 & 0.0075 \\
\hline Ccl11 & C-C motif chemokine ligand 11 & 2.06 & 0.0067 \\
\hline Ccl6 & chemokine (C-C motif) ligand 6 & 2.12 & 0.0056 \\
\hline \multicolumn{4}{|c|}{ BP: Chronic inflammatory response, $p=7.28 \times 10^{-4}$} \\
\hline Ccl11 & C-C motif chemokine ligand 11 & 2.06 & 0.0067 \\
\hline$V n n 1$ & vanin 1 & 2.10 & 0.0018 \\
\hline
\end{tabular}


Table 4. Differentially expressed genes from biological processes (BP) identified by Gene set enrichment analysis (GSEA) in SHR rats treated with $1 \% \mathrm{NaCl}$ drinking solution versus control SHR rats drinking tap water.

\begin{tabular}{|c|c|c|c|}
\hline Symbol & Name & $\log F C$ & Adjusted $p$ Value \\
\hline \multicolumn{4}{|c|}{ BP: Keratinocyte differentiation, epidermis development, $p=1.69 \times 10^{-7}$} \\
\hline$L c 1 m$ & late cornified envelope $1 \mathrm{M}$ & 1.75 & 0.025 \\
\hline Lce1f & late cornified envelope $1 \mathrm{~F}$ & 1.62 & 0.036 \\
\hline Krt1 & keratin 1 & 2.76 & 0.000003 \\
\hline Krt10 & keratin 10 & 2.98 & 0.000013 \\
\hline Lce1l & late cornified envelope $1 \mathrm{~L}$ & 2.29 & 0.00015 \\
\hline Krtdap & keratinocyte differentiation associated protein & 1.32 & 0.040 \\
\hline \multicolumn{4}{|c|}{ BP: Negative regulation of peptidase activity, $p=2.85 \times 10^{-4}$} \\
\hline Serpinb3a & serine (or cysteine) peptidase inhibitor & 4.24 & 0.000027 \\
\hline Spli & secretory leukocyte peptidase inhibitor & 1.02 & 0.036 \\
\hline Slpil3 & antileukoproteinase-like 3 & 3.02 & 0.0015 \\
\hline \multicolumn{4}{|c|}{ BP: Neutrophil chemotaxis, $p=2.77 \times 10^{-6}$} \\
\hline$I l 1 b$ & interleukin 1 , beta & 1.93 & 0.011 \\
\hline$I l 36 g$ & interleukin 36, gamma & 2.50 & 0.0024 \\
\hline Il36rn & interleukin 36 receptor antagonist & 1.45 & 0.040 \\
\hline Cxcr2 & C-X-C motif chemokine receptor 2 & 1.56 & 0.013 \\
\hline Ccl22 & C-C motif chemokine ligand 22 & 1.87 & 0.0072 \\
\hline Il36b & interleukin 36 , beta & 1.81 & 0.031 \\
\hline \multicolumn{4}{|c|}{ BP: Establishment of skin barrier, $p=1.83 \times 10^{-4}$} \\
\hline Alox $12 b$ & arachidonate 12-lipoxygenase, $12 \mathrm{R}$ type & 2.43 & 0.0028 \\
\hline Krt1 & keratin 1 & 2.76 & 0.000003 \\
\hline Flg2 & filaggrin family member 2 & 2.87 & 0.00002 \\
\hline \multicolumn{4}{|c|}{ BP: Negative regulation of endopeptidase activity, $p=6.44 \times 10^{-6}$} \\
\hline Serpinb12 & serpin family B member 12 & 2.98 & 0.000004 \\
\hline Stfa3 & stefin A3 & 2.99 & $2.89 \times 10^{-10}$ \\
\hline Serpina12 & serpin family A member 12 & 2.12 & 0.0029 \\
\hline Stfa2 & stefin A2 & 2.47 & 0.00017 \\
\hline \multicolumn{4}{|c|}{ BP: Regulation of signaling receptor activity, $p=9.08 \times 10^{-4}$} \\
\hline Btc & betacellulin & 3.39 & 0.00058 \\
\hline$I l 1 b$ & interleukin 1 , beta & 1.93 & 0.011 \\
\hline$I l 36 g$ & interleukin 36, gamma & 2.50 & 0.0024 \\
\hline Il36rn & interleukin 36 receptor antagonist & 1.45 & 0.040 \\
\hline Il18 & interleukin 18 & 1.96 & 0.013 \\
\hline Ccl22 & $\mathrm{C}-\mathrm{C}$ motif chemokine ligand 22 & 1.87 & 0.0072 \\
\hline$I l 36 b$ & interleukin 36 , beta & 1.81 & 0.031 \\
\hline
\end{tabular}




\section{Discussion}

In the current study, we found that salt sensitive SHR rats after salt loading accumulated significantly higher content of osmotically inactive $\mathrm{Na}^{+}$mainly in the skin and exhibited skin capillary rarefaction when compared to normotensive salt resistant BN- $L x$ rats. Recent paper by Rossitto et al. [15] reported that salt loading ( $1 \% \mathrm{NaCl}$ solution for 3 weeks) of SHRSP (SHR stroke-prone) rats resulted in parallel increase in skin $\mathrm{Na}^{+}$and water accumulation while no such increases were observed in normotensive salt resistant WKY rats. This result is different from previously published findings by Titze et al. $[4,16,17]$ who found significant accumulation of osmotically inactive $\mathrm{Na}^{+}$in the skin of different models of salt sensitive hypertension. It also differs from results of the current study since we detected no significant differences in tissue water accumulation between SHR and $\mathrm{BN}-L x$ rats and between treatment with tap water or $1 \% \mathrm{NaCl}$ solution. Water content in the skin: BN- $L x$ control $55.8 \%$, BN- $L x$ after salt $56.0 \%$, SHR control $55.5 \%$, SHR after salt $55.4 \%$; water content in the carcass: BN- $L x$ control $68.8 \%$, BN- $L x$ after salt $68.4 \%$, SHR control $67.2 \%$, SHR after salt $67.9 \%$ (calculated from data shown in Table 1 ). These differences could be explained by differences in measurements of tissue water and electrolyte concentrations. For estimation of $\mathrm{Na}^{+}$and water content Rossitto et al. used relatively small skin samples and other tissues samples that were digested in $\mathrm{HNO}_{3}$, while we and Titze et al. $[4,16,17]$ measured electrolyte and water content in the whole skin and carcass and used desiccation and ashing before electrolyte measurement. An additional explanation for the discrepant results could be related to differences in the rat strains studied.

The SHR rats ingested much more salt solution when compared to BN- $L x$ rats and exhibited significantly increased cumulative $\mathrm{Na}^{+}$balance. These results suggest that increased $\mathrm{Na}^{+}$accumulation in the skin of SHR rats might be due to increased salt ingestion. It has been reported that the SHR ingests significantly more salt when compared to normotensive WKY (Wistar Kyoto) rats. For instance, in the study by Chrysant et al. [18] the intake of 1\% $\mathrm{NaCl}$ solution was almost 4 times higher when compared to WKY rats. Similar findings were reported by other investigators [19-21].

A number of studies reported blood capillary rarefaction in the established stage of hypertension in many animal models including the SHR and it was suggested that capillary rarefaction can contribute to increase of total peripheral resistance [22,23]. Experiments in rats have demonstrated that salt intake has a direct effect on microvascular circulation and may be independent of blood pressure. For example, a rapid loss of microvessels in cremaster muscle occurred in Sprague-Dawley rats with reduced renal mass and hypertension but also in normotensive sham operated controls on chronic (4-6-week) as well as acute (3 days) high salt diet [22,24]. It is possible that salt, rather than working through blood pressure, may have a direct effect on microvascular network and could be one of the underlying causes for the microvascular rarefaction that occurs in hypertension.

Skin capillary rarefaction was also found in humans with essential hypertension when compared to normotensive controls [25]. Although measurement of skin capillary density in humans was used as a surrogate for capillary density in other organs these results suggest that salt sensitive hypertension in humans is associated with blood capillary rarefaction in the skin. He et al. [10] reported that in hypertensive humans, a modest reduction in salt intake increased dermal capillary density. It was also suggested that capillary rarefaction was likely to be the primary vascular abnormality that is not secondary to high blood pressure [9].

$B N-L x$ rats treated with salt showed significantly increased blood capillary density in the skin which was associated with significantly increased expression of genes regulating angiogenesis including Ereg (epiregulin), Vegfd (vascular endothelial growth factor D), Sfrp2 (secreted frizzle-related protein 2), Angpt4 (angiopoietin 4) and other genes from Positive regulation of angiogenesis and Positive regulation of endothelial cell proliferation biological processes (Table 3). Keratinocytes are able to produce several complement components. The role of complement in angiogenesis has been reviewed recently [26], however, its role in regulating skin capillary density needs further studies. Additional 
genes with significantly increased expression after treatment of BN- $L x$ rats with salt might be implicated in angiogenesis, including Ccl11, Ccr1 or Fn1.

On the other hand, SHR after salt loading showed no significant change in blood capillary density which was associated with increased dermal expression of genes regulating Keratinocyte differentiation, epidermis development and Negative regulation of peptidase activity including serpins (Serpinb3a, Serpinb12, Serpina12) which are known inhibitors of angiogenesis [27]. Osmotic stress is a potent inflammatory stimulus by triggering proinflammatory cytokine release and inflammation and also has antiangiogenic effects. During the inflammatory processes in the skin, keratinocytes act as immuno-modulators, managing inflammation via a rigorously coordinated network of inflammatory cascades [28]. Activated keratinocytes switch from their inactive status to a migratory, proliferative and pro-inflammatory phenotype and release cytokines (interleukins) and chemokines and chemokine receptors. As can be seen in Tables 3 and 4, BN-Lx skin upon salt treatment expressed genes coding for CCL11 pro-angiogenic chemokine while the SHR expresses prolinflammatory IL-1b and IL-36g cytokines. There is considerable evidence implicating also Serpinb3a in skin inflammation [29].

In the current study, we observed no significant strain and salt effects on lymph capillary density in BN-Lx and SHR rats (Figure 2B). Machnik et al. [30] reported that treatment of Sprague-Dawley rats with $1 \% \mathrm{NaCl}$ solution for drinking resulted in higher mean arterial pressure by $20 \mathrm{mmHg}$, in higher skin $\mathrm{Na}^{+}$concentrations and increased lymph capillary but not blood capillary number in the ear skin. In another paper, Machnik et al. [31] reported that Sprague-Dawley rats were salt resistant (did not increased blood pressure after $1 \% \mathrm{NaCl}$ solution for drinking) and exhibited significantly increased lymph capillary density in the ear skin after salt treatment. In addition, we observed no significant differences in dermal expression of Vegfc, Nfat5 (TonEBP), Tnfa or Nos3 (eNOS) genes between $B N-L x$ and SHR strains on normal and high salt intake. These genes were implicated in interstitial electrolyte clearance via TONEBP and VEGFC/VEGFR3-mediated modification of cutaneous lymphatic capillary function [32]. These discrepant results could be explained by differences in genetic backgrounds of SHR and BN- $L x$ versus Sprague-Dawley rats. In addition, we measured capillary density in the skin from the back of rats, not in the ear skin and blood flow might not be uniform over the entire skin surface [33].

Results of the current study suggest a possible connection of skin $\mathrm{Na}^{+}$accumulation with reduced angiogenesis in the pathogenesis of salt sensitive hypertension in the SHR. Hypertonic interstitial $\mathrm{Na}^{+}$overload in the skin, which harbors most of the body's resistance vessels, may lead to skin blood capillary rarefaction and endothelial dysfunction which together result in increased peripheral resistance and thus contribute to the pathogenesis of salt sensitivity (Figure 5). It is possible that both reduced vasodilatation (vasodysfunction) and capillary rarefaction in the SHR after salt loading could be determined by the same pathway, for instance by decreased availability of NO. NO is known to stimulate both vasorelaxation and angiogenesis. Hypothetically it might be possible that after salt loading the first response would be rapid NO induced vasorelaxation and opening of closed capillaries that would be followed by NO induced angiogenesis.

Significant differences between BN-Lx and SHR strains in salt sensitivity, skin $\mathrm{Na}^{+}$ accumulation and blood capillary density suggest strong genetic effects. The BXH/HXB recombinant inbred (RI) strains, derived from BN-Lx and SHR progenitors, show significant heritability of salt sensitivity of blood pressure [34]. Accordingly, testing the RI strains for salt effects on skin blood and lymph capillary density and on skin electrolyte concentration will enable linkage and correlation analyses with blood pressure salt sensitivity and thus identification of responsible pathophysiological mechanisms. 


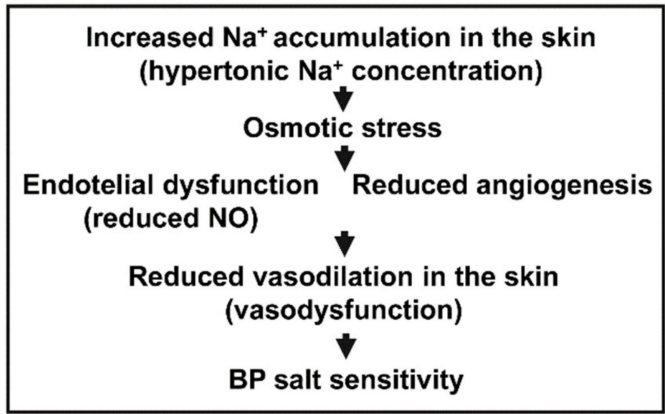

Figure 5. The proposed sequence of events showing the role of $\mathrm{Na}^{+}$accumulation in the pathogenesis of salt sensitive hypertension. According to the vasodysfunction theory $[35,36]$ the normal response to salt loading in salt resistant controls is vasodilation and a decrease in systemic vascular resistance. The failure to normally vasodilate and reduce systemic vascular resistance in the skin might be caused by skin capillary rarefaction and/or by endothelial dysfunction.

\section{Conclusions}

Results of the current study showed that salt-sensitive hypertension in the SHR is associated with increased storage of osmotically inactive $\mathrm{Na}^{+}$in the skin and skin capillary rarefaction when compared to normotensive, salt-resistant BN- $L x$ rats. Since the skin harbors most of the body's resistance vessels it is possible that blood capillary rarefaction may lead to increased peripheral resistance and thus represent new mechanism of salt sensitivity in the SHR.

Supplementary Materials: The following supporting information can be downloaded at: https:// www.mdpi.com/article/10.3390/biomedicines10020376/s1, Figure S1: Principal component analysis based on expression of 500 genes with highest variance.

Author Contributions: Conceptualization, M.P. and J.Š.; methodology, P.M., M.Š., E.S. and F.L.; formal analysis, M.P. and J.K.; writing — original draft preparation, M.P.; writing-review and editing, M.P. and J.Š.; funding acquisition, M.P. All authors have read and agreed to the published version of the manuscript.

Funding: This research was funded by Academic premium (Praemium academiae) AP1502 grant from the Czech Academy of Sciences.

Institutional Review Board Statement: The animal study protocol was approved by the Ethics Committee of Institute of Physiology, Czech Academy of Sciences, Prague (protocol code 71/2015).

Informed Consent Statement: Not applicable.

Data Availability Statement: Gene expression data are available in ArrayExpress database (accession number E-MTAB-11355).

Acknowledgments: We thank Alena Musilová for technical assistance.

Conflicts of Interest: The authors declare no conflict of interest.

\section{References}

1. Weinberger, M.H. Salt sensitivity of blood pressure in humans. Hypertension 1996, 27, 481-490. [CrossRef] [PubMed]

2. Kotchen, T.A.; Allen, W.; Cowley, A.W., Jr.; Frohlich, E.D. Salt in health and disease-A delicate balance. N. Engl. J. Med. 2016, 68, 1229-1237. [CrossRef]

3. Barba, G.; Galletti, F.; Cappuccio, F.P.; Siani, A.; Venezia, A.; Versiero, M.; Della Valle, E.; Sorrentino, P.; Tarantino, G.; Farinaro, E.; et al. Incidence of hypertension in individuals with different blood pressure salt-sensitivity: Results of a 15-year follow-up study. J. Hypertens. 2007, 25, 1465-1471. [CrossRef] [PubMed]

4. Titze, J.; Rittweger, J.; Dietsch, P.; Krause, H.; Schwind, K.H.; Engelke, K.; Lang, R.; Kirsch, K.A.; Luft, F.C.; Hilgers, K.F. Hypertension, sodium retention, calcium excretion and osteopenia in Dahl rats. J. Hypertens. 2004, 22, 803-810. [CrossRef] [PubMed] 
5. Kopp, C.; Linz, P.; Dahlmann, A.; Hammon, M.; Jantsch, J.; Müller, D.N.; Schmieder, R.E.; Cavallaro, A.; Eckardt, K.U.; Uder, M.; et al. 23Na magnetic resonance imaging-determined tissue sodium in healthy subjects and hypertensive patients. Hypertension 2013, 61, 635-640. [CrossRef]

6. Titze, J.; Dahlmann, A.; Lerchl, K.; Kopp, C.; Rakova, N.; Schröder, A.; Luft, F.C. Spooky sodium balance. Kidney Int. 2014, 85, 759-767. [CrossRef]

7. Prasad, A.; Dunnill, G.S.; Mortimer, P.S.; MacGregor, G.A. Capillary rarefaction in the forearm skin in essential hypertension. J. Hypertens. 1995, 13, 265-268. [CrossRef]

8. Antonios, T.F.; Singer, D.R.; Markandu, N.D.; Mortimer, P.S.; MacGregor, G.A. Rarefaction of skin capillaries in borderline essential hypertension suggests an early structural abnormality. Hypertension 1999, 34, 655-658. [CrossRef]

9. Antonios, T.F.; Rattray, F.M.; Singer, D.R.; Markandu, N.D.; Mortimer, P.S.; MacGregor, G.A. Rarefaction of skin capillaries in normotensive offspring of individuals with essential hypertension. Heart 2003, 89, 175-178. [CrossRef]

10. He, F.J.; Marciniak, M.; Markandu, N.D.; Antonios, T.F.; MacGregor, G.A. Effect of modest salt reduction on skin capillary rarefaction in white, black, and Asian individuals with mild hypertension. Hypertension 2010, 56, 253-259. [CrossRef] [PubMed]

11. Churchill, P.C.; Churchill, M.C.; Bidani, A.K.; Griffin, K.A.; Picken, M.; Pravenec, M.; Kren, V.; St Lezin, E.; Wang, J.M.; Wang, N.; et al. Genetic susceptibility to hypertension-induced renal damage in the rat. Evidence based on kidney-specific genome transfer. J. Clin. Investig. 1997, 100, 1373-1382. [CrossRef] [PubMed]

12. Liška, F.; Mancini, M.; Krupková, M.; Chylíková, B.; Křenová, D.; Šeda, O.; Šilhavý, J.; Mlejnek, P.; Landa, V.; Zídek, V.; et al. Plzf as a candidate gene predisposing the spontaneously hypertensive rat to hypertension, left ventricular hypertrophy, and interstitial fibrosis. Am. J. Hypertens. 2014, 27, 99-106. [CrossRef] [PubMed]

13. Liška, F.; Landa, V.; Zídek, V.; Mlejnek, P.; Šilhavý, J.; Šimáková, M.; Strnad, H.; Trnovská, J.; Škop, V.; Kazdová, L.; et al Downregulation of Plzf gene ameliorates metabolic and cardiac traits in the spontaneously hypertensive rat. Hypertension 2017, 69, 1084-1091. [CrossRef]

14. Lunova, M.; Kubovciak, J.; Smolková, B.; Uzhytchak, M.; Michalova, K.; Dejneka, A.; Strnad, P.; Lunov, O.; Jirsa, M. Expression of interferons lambda 3 and 4 induces identical response in human liver cell lines depending exclusively on canonical signaling. Int. J. Mol. Sci. 2021, 22, 2560. [CrossRef] [PubMed]

15. Rossitto, G.; Mary, S.; Chen, J.Y.; Boder, P.; Chew, K.S.; Neves, K.B.; Alves, R.L.; Montezano, A.C.; Welsh, P.; Petrie, M.C.; et al. Tissue sodium excess is not hypertonic and reflects extracellular volume expansion. Nat. Commun. 2020, 11, 4222. [CrossRef] [PubMed]

16. Titze, J.; Lang, R.; Ilies, C.; Schwind, K.H.; Kirsch, K.A.; Dietsch, P.; Luft, F.; Hilgers, K.F. Osmotically inactive skin Na+ storage in rats. Am. J. Physiol. Ren. Physiol. 2003, 285, F1108-F1117. [CrossRef]

17. Titze, J.; Bauer, K.; Schafflhuber, M.; Dietsch, P.; Lang, R.; Schwind, K.H.; Luft, F.C.; Eckardt, K.-U.; Hilgers, K.F. Internal sodium balance in DOCA-salt rats: A body composition study. Am. J. Physiol. Ren. Physiol. 2005, 289, F793-F802. [CrossRef]

18. Chrysant, S.G.; Walsh, G.M.; Kem, D.C.; Frohlich, E.D. Hemodynamic and metabolic evidence of salt sensitivity in spontaneously hypertensive rats. Kidney Int. 1979, 15, 33-37. [CrossRef]

19. Flynn, F.W.; Culver, B.; Newton, S.V. Salt intake by normotensive and spontaneously hypertensive rats: Two-bottle and lick rate analyses. Physiol. Behav. 2003, 78, 689-696. [CrossRef]

20. McConnell, S.D.; Henkin, R.I. Na,Cl preference in spontaneously hypertensive rats; age and blood pressure effects. Am. J. Physiol. 1973, 225, 624-627. [CrossRef]

21. Di Nicolantonio, R.; Kren, V.; Zidek, V.; Pravenec, M. Salt preference of congenic strains derived from the spontaneously hypertensive rat. Physiol. Behav. 2004, 80, 617-622. [CrossRef]

22. Hansen-Smith, F.M.; Morris, L.W.; Greene, A.S.; Lombard, J.H. Rapid microvessel rarefaction with elevated salt intake and reduced renal mass hypertension in rats. Circ. Res. 1996, 79, 324-330. [CrossRef] [PubMed]

23. Greene, A.S.; Tonellato, P.J.; Lui, J.; Lombard, J.H.; Cowley, A.W., Jr. Microvascular rarefaction and tissue vascular resistance in hypertension. Am. J. Physiol. 1989, 256 Pt 2, H126-H131. [CrossRef] [PubMed]

24. Greene, A.S.; Lombard, J.H.; Cowley, A.W., Jr.; Hansen-Smith, F.M. Microvessel changes in hypertension measured by Griffonia simplicifolia I lectin. Hypertension 1990, 15 Pt 2, 779-783. [CrossRef]

25. Triantafyllou, A.; Anyfanti, P.; Pyrpasopoulou, A.; Triantafyllou, G.; Aslanidis, S.; Douma, S. Capillary rarefaction as an index for the microvascular assessment of hypertensive patients. Curr. Hypertens. Rep. 2015, 17, 33. [CrossRef] [PubMed]

26. Markiewski, M.M.; Daugherity, E.; Reese, B.; Karbowniczek, M. The role of complement in angiogenesis. Antibodies 2020, 9, 67. [CrossRef]

27. Silverman, G.A.; Whisstock, J.C.; Bottomley, S.P.; Huntington, J.A.; Kaiserman, D.; Luke, C.J.; Pak, S.C.; Reichhart, J.-M.; Bird, P.I Serpins flex their muscle: I. Putting the clamps on proteolysis in diverse biological systems. J. Biol. Chem. 2010, 285, 24299-24305. [CrossRef] [PubMed]

28. Juráňová, J.; Franková, J.; Ulrichová, J. The role of keratinocytes in inflammation. J. Appl. Biomed. 2017, 15, 169-179. [CrossRef]

29. Sivaprasad, U.; Kinker, K.G.; Ericksen, M.B.; Lindsey, M.; Gibson, A.M.; Bass, S.A.; Hershey, N.S.; Deng, J.; Medvedovic, M.; Hershey, G.K.K. SERPINB3/B4 contributes to early inflammation and barrier dysfunction in an experimental murine model of atopic dermatitis. J. Investig. Derm. 2015, 135, 160-169. [CrossRef] [PubMed] 
30. Machnik, A.; Neuhofer, W.; Jantsch, J.; Dahlmann, A.; Tammela, T.; Machura, K.; Park, J.K.; Beck, F.X.; Müller, D.N.; Derer, W.; et al. Macrophages regulate salt-dependent volume and blood pressure by a vascular endothelial growth factor-C-dependent buffering mechanism. Nat. Med. 2009, 15, 545-552. [CrossRef]

31. Machnik, A.; Dahlmann, A.; Kopp, C.; Goss, J.; Wagner, H.; van Rooijen, N.; Eckardt, K.U.; Müller, D.N.; Park, J.K.; Luft, F.C.; et al. Mononuclear phagocyte system depletion blocks interstitial tonicity-responsive enhancer binding protein/vascular endothelial growth factor $C$ expression and induces salt-sensitive hypertension in rats. Hypertension 2010, 55, 755-761. [CrossRef]

32. Wiig, H.; Schröder, A.; Neuhofer, W.; Jantsch, J.; Kopp, C.; Karlsen, T.V.; Boschmann, M.; Goss, J.; Bry, M.; Rakova, N.; et al. Immune cells control skin lymphatic electrolyte homeostasis and blood pressure. J. Clin. Investig. 2013, 123, 2803-2815. [CrossRef] [PubMed]

33. Rendell, M.S.; Finnegan, M.F.; Healy, J.C.; Lind, A.; Milliken, B.K.; Finney, D.E.; Bonner, R.F. The relationship of laser-Doppler skin blood flow measurements to the cutaneous microvascular anatomy. Microvasc. Res. 1998, 55, 3-13. [CrossRef] [PubMed]

34. Pravenec, M.; Klír, P.; Kren, V.; Zicha, J.; Kunes, J. An analysis of spontaneous hypertension in spontaneously hypertensive rats by means of new recombinant inbred strains. J. Hypertens. 1989, 7, 217-221. [CrossRef]

35. Kurtz, T.W.; DiCarlo, S.E.; Pravenec, M.; Schmidlin, O.; Tanaka, M.; Morris, R.C., Jr. An alternative hypothesis to the widely held view that renal excretion of sodium accounts for resistance to salt-induced hypertension. Kidney Int. 2016, 90, 965-973. [CrossRef] [PubMed]

36. Kurtz, T.W.; Dicarlo, S.E.; Pravenec, M.; Morris, R.C., Jr. The pivotal role of renal vasodysfunction in salt sensitivity and the initiation of salt-induced hypertension. Curr. Opin. Nephrol. Hypertens. 2018, 27, 83-92. [CrossRef] [PubMed] 